\title{
Carcinomatosis peritoneal. Quimioterapia intraperitoneal con hipertermia
}

\author{
J. Farré Alegre, P. Bretcha Boix, M. L. Martín, M. Sureda, A. Brugarolas
}

\section{Introducción}

La diseminación peritoneal constituye uno de los principales patrones de recaída tras cirugía en los tumores de localización abdominal y pélvica. Ha sido considerada clásicamente como un signo de enfermedad generalizada, tratada de forma paliativa y de desenlace inevitablemente fatal. En un reciente estudio multicéntrico en el que se incluían pacientes con carcinomatosis de origen no ginecológico, la supervivencia media era de 3.1 meses $^{1}$. En estudios en los que se ha tratado con quimioterapia sistémica, se han obtenido buenas respuestas tumorales, aunque sin mejoría en la supervivencia.

La diseminación de las células tumorales en el peritoneo puede ser espontánea o producirse durante la cirugía, por mecanismos como la formación de émbolos tumorales por presión, la fuga de células malignas al cortar los vasos linfáticos o la siembra de las mismas en la cavidad peritoneal durante la disección quirúrgica. Va seguida habitualmente de la invasión o perforación de la serosa.

Una vez extirpado el tumor primario, los factores de crecimiento celular involucrados en la cicatrización estimulan el crecimiento de las células malignas viables atrapadas 0 en coágulos sanguíneos intraabdominales, o por la fibrina en superficies peritoneales traumatizadas. Paralelamente, el atrapamiento de dichas células dificulta el acceso a ellas de la quimioterapia sistémica, disminuyendo o anulando su eficacia $^{2,3}$.

Desde 1980 han aparecido en la literatura nuevas propuestas para el tratamiento de los pacientes afectos de diseminación tumoral peritoneal, un grupo de difícil manejo terapéutico y emocional, derivado de la perspectiva de fracaso terapéutico con perdida rápida y progresiva de la calidad de vida ${ }^{4}$. La administración de drogas antitumorales por vía intraperitoneal, la inmunoterapia intracavitaria, la terapia fotodinámica o la quimioterapia e hipertermia intraperitoneal constituyen una muestra de las líneas de trabajo en esta dirección ${ }^{4}$.

La perfusión intraperitoneal con quimioterapia e hipertermia ha ofrecido hasta el momento resultados esperanzadores. Consiste en realizar la cirugía de exéresis máxima o debulking, seguida de dosis altas de quimioterapia regional en perfusión hipertérmica durante el mismo acto operatorio. En ocasiones se completa la técnica con la administración de dosis altas de quimioterapia en la cavidad peritoneal los días posteriores a la cirugía, a través de catéteres provisionales implantados a tal efecto.

La cirugía posibilita la reducción de la enfermedad peritoneal a un tamaño mínimo y libera todas las adherencias, creando las condiciones óptimas para obtener mayor eficacia de los citostáticos. Estos son potenciados por el calor, que también tiene un efecto citotóxico por sí mismo, se elimina el atrapamiento celular, y se consiguen niveles altos de fármaco en la zona a tratar, imposibles de lograr por vía sistémica debido a su toxicidad ${ }^{5-8}$.

Debido a la barrera peritoneo-plasmática, las sustancias de alto peso molecular como mitomicina C, 5-fluorouracilo, cisplatino o doxorubicina, permanecen largo tiempo dentro del abdomen antes de su eliminación a través de la sangre, lo que las hace especialmente indicadas en esta modalidad terapéutica.

Ratio del área bajo la curva de las drogas administradas en relación a la exposición de la superficie peritoneal versus la sistémica

\begin{tabular}{lcc}
\hline Droga & Peso Molecular & Ratio AUC \\
\hline 5-Fluorouracilo & 130 & 250 \\
Mitomicina C & 334 & 75 \\
Doxorrubicina & 544 & 500 \\
Cisplatino & 300 & 20 \\
Taxol & 808 & 1000 \\
Gemcitabina & 263 & 50 \\
\hline
\end{tabular}

Plataforma Oncológica

Hospital San Jaime

Torrevieja (Alicante) 


\section{Beneficios de la quimioterapia intraperitoneal con hipertermia tras cirugía citorreductora}

- El calor tiene efectos antitumorales por sí mismo.

- El calor incrementa la citotoxicidad por sí mismo.

- El calor incrementa la penetración de los medicamentos en los tejidos.

- Se consigue una distribución uniforme de la quimioterapia intraperitoneal en todas las superficies del abdomen y la pelvis..

- Se eliminan las náuseas y los vómitos

- Durante el tiempo de hipertermia se monitorizan todos los parámetros (hemodinámicos, temperatura, coagulación, etc.)

Indicaciones de la cirugía citorreductora con quimioterapia intraperitoneal e hipertermia

- Gran volumen de carcinomatosis peritoneal no invasiva o sarcomatosis.

- Mesotelioma peritoneal.

- Bajo volumen de implantes peritoneales de cáncer invasivo.

- Cáncer gastrointestinal perforado.

- Cáncer adherido a estructuras adyacentes.

- Cáncer gastrointestinal con citología peritoneal positiva.

- Cáncer gastrointestinal con afectación del ovario.

- Rotura tumoral con diseminación durante el acto quirúrgico.

- Cáncer de ovario recurrente después de largo tiempo libre de enfermedad.

- Paliación en pacientes con ascitis carcinomatosas.

\section{Técnica del coliseum}

La cirugía citorreductora busca disminuir la enfermedad en la cavidad abdominal hasta la mínima cantidad posible. En primer lugar se evalúa la carga tumoral del paciente y se obtiene el índice de carcinomatosis (PCI), dividiendo el abdomen en trece áreas, de cero a doce, según el siguiente gráfico (tomado de www.surgicaloncology.com).

La citorreducción obtenida tras la cirugía en se considera completa (RO) cuando no hay implantes residuales macroscópicos. En los casos que persisten implantes residuales, se divide en R1 si son menores de $25 \mathrm{~mm}$ y R2 si son mayores de este tamaño. En tumores no invasivos (pseudomixoma, mesotelioma, etc) las categorías R0 y R1 se consideran equivalentes.

Al terminar el procedimiento se colocan cuatro catéteres de succión, dos subdiafragmáticos y dos pélvicos. También se coloca un catéter tipo Tenkhoff para la quimioterapia postoperatoria como línea de entrada. La temperatura central se monitoriza mediante dos termómetros intraperitoneales y otro esofágico. Se coloca un termómetro adicional para control de la temperatura a la entrada de la perfusión.

Una vez montado el separador de Thompson se suspende el abdomen del separador con dos suturas continuas a la piel de la incisión abdominal. Mediante la cobertura con un plástico se aísla la cavidad, con un cierre en forma de campana, en cuyo interior se coloca un aspirador de humos que recogerá los vapores generados durante la perfusión con el quimioterápico. Un orificio en dicha cobertura permitirá al cirujano, equipado con guantes especiales, el acceso y control manual de la distribución de los fluídos por el interior de toda la cavidad abdominal (Foto 1).

La perfusión con hipertermia se hace durante 90 minutos con dos bombas de extracorpórea y un equipo de tubos y

\section{Peritoneal Cancer Index}

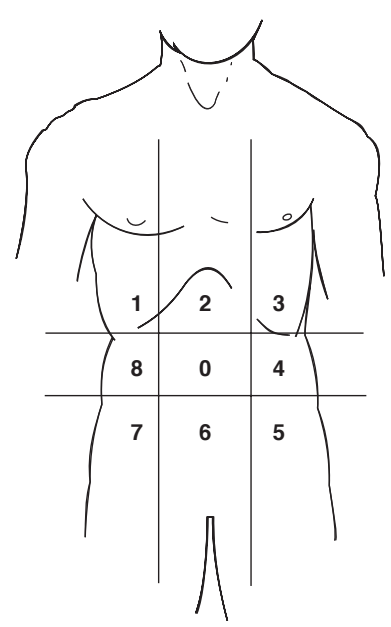

Regions

0 Central

1 Right Upper

2 Epigastrium

3 Left Upper

4 Left Flank

5 Left Lower

6 Pelvis

7 Right Lower

8 Right Flank

9 Upper Jejunum

10 Lower Jejunum

11 Upper Ileum

12 Lower Ileum

\section{$\mathrm{PCl}$}

PCI MEDIO: 4.25 (rango: 0 - 24)
Lesion Size Score

\section{Lesion Size Score}

LS 0 No tumor seen

LS 1 Tumor up to $05 \mathrm{~cm}$.

LS 2 Tumor up to $50 \mathrm{~cm}$.

LS 3 Tumor $50 \mathrm{~cm}$.

or confluence

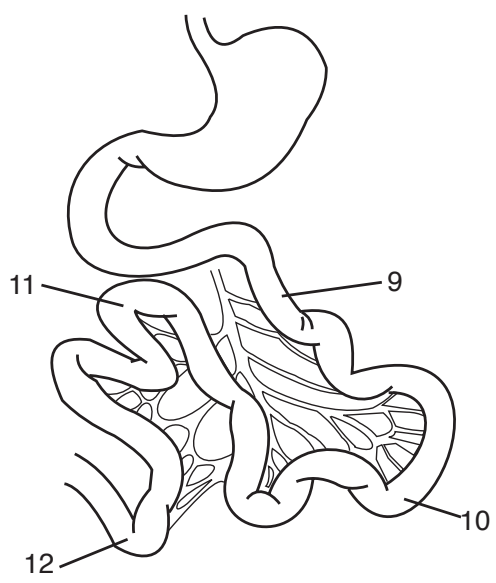




\section{J. Farré Alegre y cols.}

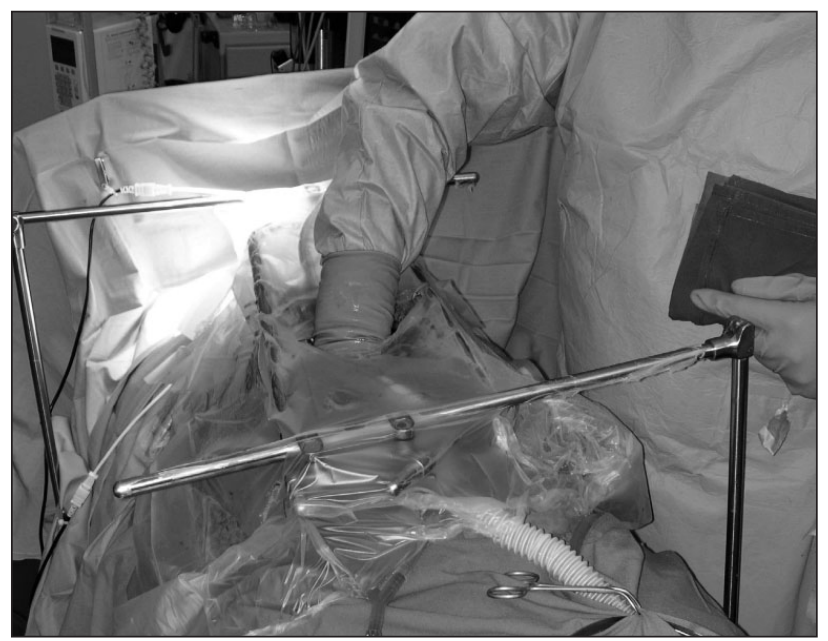

Foto 1 .

reservorio de cardiotomía. El flujo de la perfusión oscila entre 500 y $1000 \mathrm{ml} /$ minuto, con una solución de dextrosa al $1.5 \%$ (entre 3 y 5 litros) a los que se añade el citostático. El circuito pasa por intercambiador de calor para conseguir una temperatura en catéter de entrada de 43-44 grados. Durante todo el procedimiento debe haber un especial control y monitorización de las constantes del paciente, buscando mantener diuresis de unos $400 \mathrm{ml} / \mathrm{h}$ (ver gráfico. Reproducido de www.surgicaloncology.com).

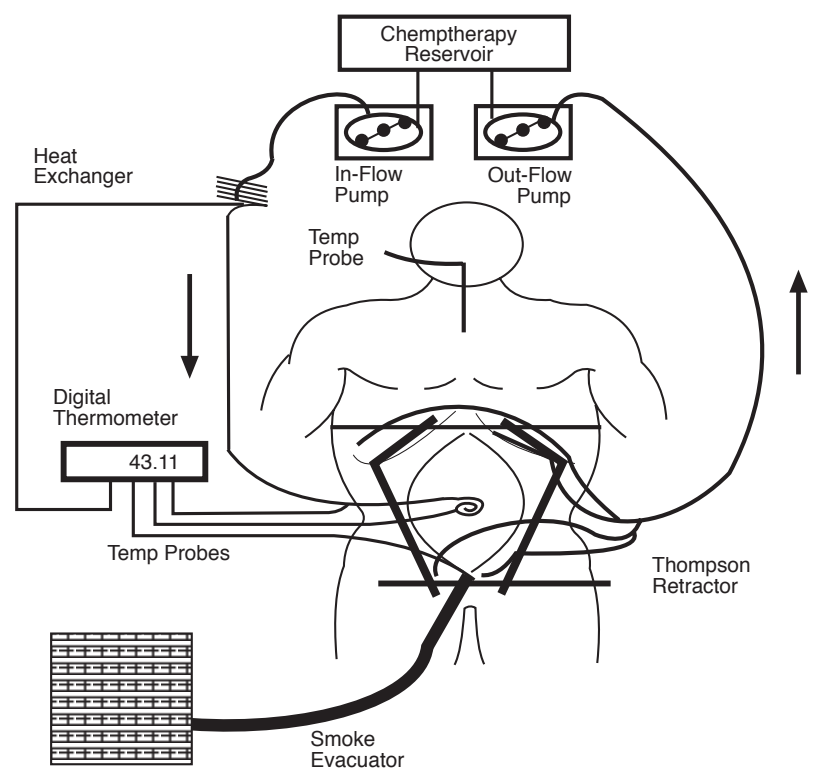

Los procesos de reconstrucción y suturas se hacen cuando termina la perfusión.

\section{Experiencia en el hospital San Jaime}

Entre Junio de 2001 y Setiembre de 2003 se trataron en el Hospital San Jaime 24 pacientes (20 mujeres y 4 varones), con una edad media de 55,5 años (25-78 años). Los tumores primarios fueron: cáncer colorectal (6 casos), pseudomixoma peritoneal (2), cáncer gástrico (1), cáncer de ovario (13) y cáncer de endometrio (2). En 6 casos de cáncer de ovario (todos ellos estadio III-IV en el momento del diagnóstico) se indicó la técnica como tratamiento de consolidación tras haber recibido la quimioterapia sistémica adyuvante.

Cuatro pacientes presentaron la carcinomatosis de forma sincrónica y 20 de forma metacrónica (diagnosticados durante el seguimiento de un tumor abdominal previamente resecado). Un paciente presentó metástasis hepáticas que fueron tratadas mediante ablación con radiofrecuencia en el mismo acto quirúrgico.

Al final de la cirugía de exéresis, en 22 pacientes se consiguieron resecciones $\mathrm{RO} \circ \mathrm{R} 1$. En los 2 pacientes restantes la resección fue $\mathrm{R} 2$. El índice de carcinomatosis medio fue de $4,25(0-24)$.

En los casos de adenocarcinoma de origen apendicular, colorrectal y gástrico, así como en el pseudomixoma peritoneal se utilizó para la perfusión mitomicina $C$, con una dosis para varones de $12,5 \mathrm{mg} / \mathrm{m}^{2}$ (máximo 25) y para mujeres de $10 \mathrm{mg} / \mathrm{m}^{2}$. Durante los 5 primeros días del postoperatorio se administró por vía intraperitoneal 5-FU a una dosis de $15 \mathrm{mg} / \mathrm{kg} /$ día. En el caso de cáncer de ovario inicialmente se utilizó taxol $\left(60 \mathrm{mg} / \mathrm{m}^{2}\right)$, seguido del mismo esquema de 5-FU.

No hubo mortalidad intraoperatoria y se completó el procedimiento en todos los casos. El tiempo medio de todo el procedimiento fue de 6,36 horas s-8, $^{5}$.

Todos los pacientes permanecieron en la Unidad de Cuidados Intensivos durante, como mínimo, los 5 días que duraba la quimioterapia peritoneal postoperatoria. La morbilidad global observada fue del $46 \%(11 / 24)$, distribuídos en ileo paralítico prolongado (6 casos), abscesos de pared (2), hemoperitoneo (1), dehiscencia anastomótica (1) y pielonefritis aguda por $E$. coli (1).

\section{Supervivencia actuarial}

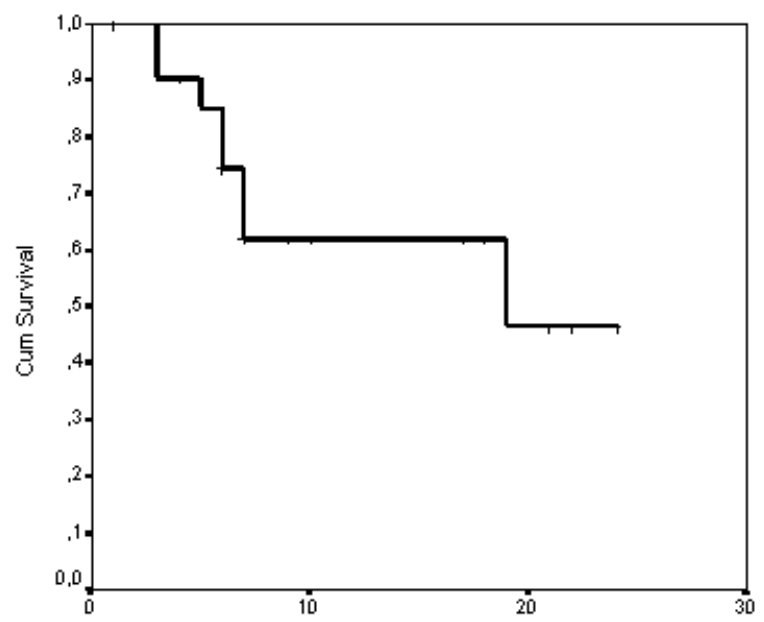

Periodo libre de progresión (meses) 
Hubo un caso de mortalidad postoperatoria a los 21 días de la intervención. Se trataba de una mujer de 47 años, diagnosticada e intervenida por un adenocarcinoma gástrico dos años antes, que presentaba una recidiva a nivel del mesocolon transverso. Se practicó una hemicolectomia derecha ampliada, resección de una asa intestinal, colecistectomía, anexectomía bilateral y perfusión intraperitoneal con mitomicina $\mathrm{C}$ e hipertermia. La causa de la muerte fue peritonitis y sepsis por dehiscencia anastomótica.

La estancia media hospitalaria ha sido de 17,8 días (1048). Una vez realizada el alta hospitalaria el seguimiento clínico, analítico y radiológico se realizó de forma mensual.

La mediana de seguimiento ha sido de 11 meses (2-26). La supervivencia actuarial libre de enfermedad según el método de Kaplan y Meier ha sido del $46 \%$. En el período referido ha habido, además de la comentada, otra muerte por infarto de miocardio a los tres meses de la intervención.

Se han objetivado 7 recidivas en el mismo período. Una paciente de 33 años tratada por una carcinomatosis de origen colónico, presentó una recidiva presacra a los 6 meses de la intervención. Fue rescatada quirúrgicamente y se encuentra sin evidencia de enfermedad 17 meses después. Otra paciente tratada inicialmente por una carcinomatosis de origen ovárico presentó una metástasis hepática única a los 7 meses. Fue tratada mediante ablación por radiofrecuencia, permaneciendo libre de enfermedad 10 meses después. Los cinco pacientes restantes están actualmente recibiendo quimioterapia sistémica.

\section{Conclusiones}

La carcinomatosis peritoneal ha sido considerada como una entidad fatal y clásicamente tratada de forma paliativa.

Spratt y cols (1970) fueron los primeros en utilizar la quimioterapia con hipertermia en estudios experimentales con el objetivo de optimizar el efecto citotóxico de las drogas. En 1979 se realizó la primera quimioterapia intraperitoneal con hipertermia en un paciente portador de un pseudomixoma peritoneal después de cirugía citorreductora7. Desde 1982, el Dr PH Sugarbaker planteó la diseminación peritoneal de ciertos cánceres como un estadío locoregional de la enfermedad y desarrolló una alternativa terapéutica basada en el tratamiento quirúrgico de la enfermedad macroscópica peritoneal mediante cirugía citorreductora radical seguida de quimioterapia intraperitoneal con hipertermia para tratar la enfermedad microscópica residual. Con este planteamiento terapéutico se obtuvieron supervivencias a 5 años del $30 \%$ y $50 \%$ en grupos seleccionados de pacientes que hasta entonces eran considerados pacientes terminales. Resultados similares han sido comunicados por otros grupos tras la implementación de esta compleja técnica ${ }^{4,9-16}$.

Como es de esperar los mejores resultados se obtienen en aquellos casos en los que existe menor volumen de carcinomatosis y en los que la cirugía ha conseguido una buena citorreducción. Un índice de carcinomatosis superior a 13 según el esquema de Sugarbaker es sinónimode mal pronóstico. Por lo tanto el resultado está relacionado con la selección adecuada de los pacientes que pueden beneficiarse de este tratamiento.

Los efectos antineoplásicos de la quimioterapia son potenciados por el calor en virtud del aumento de la permeabili- dad celular, por alteración del transporte activo de las drogas y la alteración del metabolismo celular.

La administración de altas dosis de quimioterapia intraperitoneal es posible sin toxicidad renal ni sistémica. Al mismo tiempo se minimizan los efectos secundarios ya que el paciente permanece anestesiado y en todo momento se mantienen monitorizados todos los parámetros.

A la luz de los datos expuestos nos encontramos ante una modalidad terapéutica prometedora. La adecuada selección de pacientes, el perfeccionamiento de los procedimientos técnicos y la disponibilidad de nuevos quimioterápicos se traducirán con mucha probabilidad en una mejora sustancial de los resultados. La combinación con otras modalidades terapéuticas, locorregionales o sistémicas, abre líneas de trabajo esperanzadoras para un conjunto de pacientes que en este momento carecen de perspectivas más allá de la paliación.

\section{Bibliografía}

1. Sadeghi B, Arvieux C, Glehen O, et al. Peritoneal carcinomatosis from non gynecologic malignancies: Results from the EVOCAPE 1 multicentric prospective study. Cancer 2000; 88:358363.

2. Sugarbaker PH. Intraperitoneal chemotherapy and cytoreductive surgery for the prevention and treatment of peritoneal carcinomatosis and sarcomatosis. Semin Surg Oncol 1998; 14: 254-61.

3. Sugarbaker PH, Chang D. Results of treatment of 385 patients with peritoneal surface spread of appendiceal malignancy. Ann Surg Oncol 1999; 6:727-731.

4. Glehen O, Mithieux F, Osinsky D, Beaujard AC, Freyer G, Guertsch Ph, Francois Y, Peyrat P, Panteix G, Vignal J, Gilly F. Surgery combined with peritonectomy procedures and intraperitoneal chemohyperthermia in abdominal cancers with peritoneal carcinomatosis: a phase II study. J Clin Oncol 2003; 21(5):799-806

5. Verwall V, van Ruth $S$, de Bree E, van Slooten W, van Tinteren $\mathrm{H}$, Boot H, Zoetmulder F. Randomized trial of cytoreduction and hyperthermic intraperitoneal chemotherapy versus systemic chemotherapy and palliative surgery in patients with peritoneal carcinomatosis of colorectal cancer. J Clin Oncol 2003; 21: 3737-43.

6. Begossi G, Gonzalez Moreno S, Ortega Perez G, Fon L, Sugarbaker PH. Cytoreduction and intraperitoneal chemotherapy for the management of peritoneal carcinomatosis, sarcomatosis and mesothelioma. EJSO 2002; 28:80-87.

7. Witkamp A, de Bree E, Van Goethem, Zoetmulder F. Rationale and techniques of intraoperative hyperthermic intraperitoneal chemotherapy. Cancer Treat Rev 2001; 27:365- 374.

8. Takahashi I, Emi Y, Hasuda S, Kakeji Y, Maehara Y, Sugimachi $\mathrm{K}$. Clinical application of hyperthermia combined with anticancer drugs for the treatment of solid tumors. Surgery 2002; 131: S78-84.

9. Witkamp A, de Bree E, Kaag M, Boo H, Beijnen J, Van Slooten $G$, Van Coevorden F, Zoetmulder F. Extensive cyłoreductive surgery followed by intraoperative hyperthermic intraperitoneal chemotherpy with mitomycin- $\mathrm{C}$ in patients with peritoneal carcinomatosis of colorectal origin. Eur J Cancer 2001; 37:979-984.

10. Carmingnani C, Esquivel J, Sugarbaker PH. Cytoreductive surgery and intraperitoneal chemotherapy for the treatment of peritoneal surface malignancy. Rev Oncol 2003; 5(4):192-8. 


\section{J. Farré Alegre y cols.}

11. Sugarbaker PH. Cytoreduction including total gastrectomy for pseudomyxoma peritonei. Br J Surg 2002; 89:208-12.

12. Elias D, Bonnay M, Puizillou J, Antoun S, Demirdjian S, El Otmany A, Pignon J, Drouard-Troalen L, Ouellet J, Ducreux M. Heated intraoperative intraperitoneal oxaliplatin after complete resection of peritoneal carcinomatosis: pharmacokinetics and tissue distribution. Ann Oncol 2002; 13(2):267-272.

13. Sugarbaker $P$, Jablonski KA. Prognostic features of 51 colorectal and 130 appendiceal cancer patients with peritoneal carcinomatosis treated by cytoreductive surgery and intraperitoneal chemotherapy. Ann Surg 1995; 221:124-132.
14. Glehen O, Schreiber V, Cotte E, Sayag-Beaujard A, Osinsky D, Freyer G, Francois Y, Vignal J, Gilly F. Cytoreductive surgery and intraperitoneal chemohyperthermia for peritoneal carcinomatosis arising from gastric cancer. Arch Surg 2004; 139(1): 20-26.

15. Shen P, Levine E, Hall J, Case D, Russel G, fleming R, Mc Quellon $R$, Geisinger $K$, Loggie $B$. Factors predicting survival after intraperitoneal hyperthermic chemotherapy with mitomicin $C$ after cytoreductive surgery for patients with peritoneal carcinomatosis. Arch of Surg 2003; 138(1):26-33.

16. Jayne $D$, fook S, Loi $C$, Seow-Choen F. Peritoneal carcinomatosis from colorectal cancer. Br J Surg 2002; 89(12):1545-1550. 